\title{
Astaxanthin sources: Suitability for human health and nutrition
}

\author{
Bob Capelli" ${ }^{*}$; Shawn Talbott ${ }^{2}$; Lixin Ding ${ }^{3}$ \\ ${ }^{1}$ Algae Health Sciences, Irvine, CA, USA $2{ }_{2}^{2}$ Eqqil, Draper, UT, USA; ${ }^{3}$ BGG, Irvine, CA, USA \\ Corresponding Author: Bob Capelli, Algae Health Sciences, Irvine, CA, USA.
}

Submission Date: November $21^{\text {st }}$, 2018. Acceptance Date: June $27^{\text {th }}$, 2019. Publication Date: June $30^{\text {th }}, 2019$.

Citation: Capelli B., Talbott S., Ding L. Astaxanthin Sources: Suitability for Human Health and Nutrition. Functional Foods in Health and Disease 2019; 9(6): 430-445. DOI: https://doi.org/10.31989/ffhd.v9i6.584

\begin{abstract}
Background: Astaxanthin (AX) has been consumed as a nutritional supplement for approximately twenty years. The primary source has been a natural plant-based supplement from the single-cell alga Haematococcus pluvialis (NAT-AX). Recently, Astaxanthin from other sources has entered the marketplace. The primary alternative source in the human nutritional supplement market has been a synthetic form of Astaxanthin produced from petrochemicals (SYN-AX). Additionally, a very small amount of Astaxanthin from a genetically-manipulated yeast Xanthophyllomyces dendrorhous (former nomenclature Phaffia rhodozyma, still commonly referred to as "Phaffia") (PH-AX) is also available in some supplement products. The three forms have substantial chemical differences. In addition to the chemical differences between sources of AX, in-vitro research has demonstrated profound differences in antioxidant strength and animal research has revealed fundamental differences in health benefits. In all cases, NAT-AX has proven more biologically active than the other sources. This review is designed to assist readers in understanding which form(s) of AX are suitable for consumers desiring preventive or therapeutic health benefits.
\end{abstract}

Results: In head-to-head antioxidant experiments, NAT-AX demonstrated 14X to 90X greater antioxidant activity than SYN-AX. In numerous animal trials in diverse species, NAT-AX in esterified form has demonstrated superior efficacy in increasing lifespan; treating skin cancer; preventing the formation of gastric ulcers; improving resistance to stress; decreasing reactive oxygen species (ROS); increasing retinol conversion in the liver; augmenting enzyme levels; increasing growth rates; and improving exercise endurance.

From a safety perspective, NAT-AX has been the subject of human clinical trials demonstrating safety and a wide variety of health benefits. In addition, no documented adverse events have surfaced during its twenty years of distribution as a food supplement for humans. SYN-AX and PH-AX have not been proven safe for direct human consumption and have not demonstrated any health benefits in clinical trials. Due to these facts, SYN-AX and PH-AX have 
not been allowed for human consumption by government regulators in many countries while NATAX is widely accepted in most countries around the world.

Conclusion: Based on our review of the literature below, we recommend NAT-AX as the sole form of AX for human consumption until SYN-AX and PH-AX have been proven safe and efficacious through human clinical research.

\section{REVIEW}

AX is related to other members of the carotenoid family, a group of pigments that provide reactive oxygen species (ROS) scavenging activity in many plants and animals. AX is classified within the carotenoid family as a xanthophyll and is closely related to lutein, zeaxanthin, and canthaxanthin. Xanthophylls are structurally different from carotenes (the other group of carotenoids including beta-carotene (BC) and lycopene) due to hydroxyl groups and carbonyl groups at the end of the AX molecule. AX has more hydroxyl groups than other xanthophylls which result in enhanced antioxidant activity and increased health benefits in humans and animals. Additionally, $\mathrm{AX}$ has enone groups which extend the conjugation of $\mathrm{C}=\mathrm{C}$ bonds which make $\mathrm{AX}$ more active as an antioxidant than other carotenoids [1, 2, 3].

The wide-ranging effects of carotenoids on membranes has been hypothesized as the basis for enhanced performance of xanthophylls such as AX. BC and lycopene cause disorder of model membranes and result in lipid peroxidation; in contrast, AX preserves the structure of the model membrane [4]. Additionally, while carotenes such as BC and lycopene can become pro-oxidants under certain conditions and hasten the proliferation of ROS, AX has never shown the capacity to create a pro-oxidant effect [5].

The three different forms of $\mathrm{AX}$ in this discussion are the result of entirely dissimilar processes:

- NAT-AX is found in the highest quantities in nature in Haematococcus pluvialis, a ubiquitous uni-cellular microalgae, which grows in fresh water throughout the world. When these algae undergo environmental stress, they hyperaccumulate NAT-AX as a survival mechanism.

- SYN-AX is synthesized from petrochemicals in a highly involved, multi-step process.

- $\mathrm{PH}-\mathrm{AX}$ is produced from a species of yeast which, in nature, produces small amounts of AX. Companies involved in the commercial production of $\mathrm{PH}-\mathrm{AX}$ have genetically-manipulated this species to produce exponentially more AX [6].

\section{Chemical Differences between Forms of Astaxanthin}

There are three significant differences chemically between NAT-AX, SYN-AX, and PH-AX:

Difference \#1: NAT-AX is comprised of 95.7\% esterified AX molecules, both mono-esterified (87.0\% of the total carotenoid fraction with one fatty acid molecule attached to one end of the AX molecule) and di-esterified (8.7\% of the total carotenoid fraction with one fatty acid molecule attached to each end of the AX molecule) (Fig. 1; Fig 2). Conversely, SYN-AX and PH-AX are exclusively "free" astaxanthin (non-esterified without fatty acids attached to either end of the molecule) [7]. 


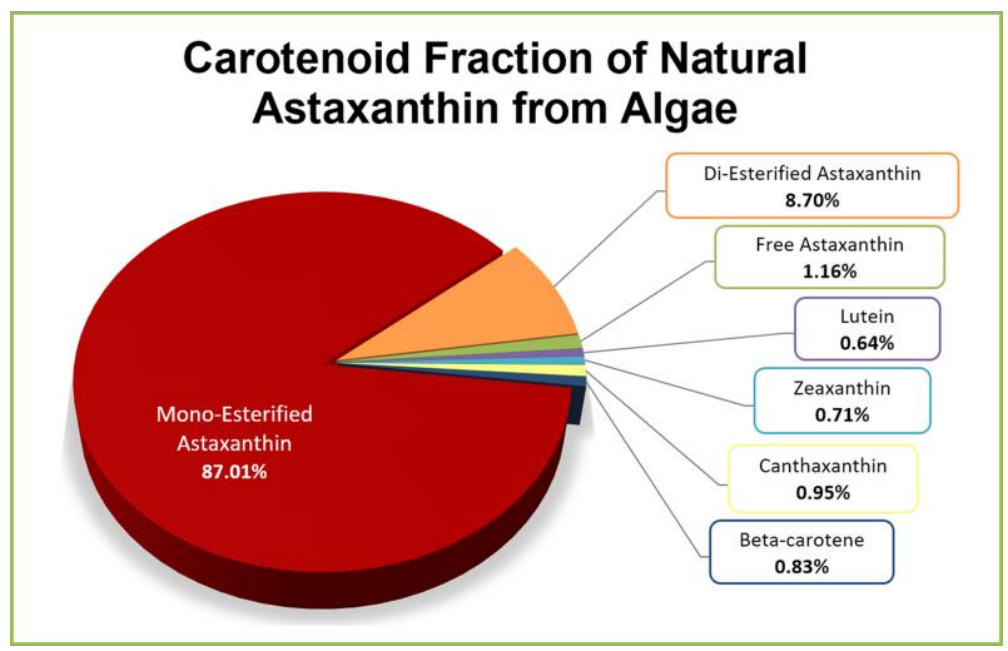

Figure 1. Natural Astaxanthin Carotenoid Fraction: Natural Astaxanthin from algae is composed primarily of esterified molecules. Approximately $95.7 \%$ of Natural Astaxanthin molecules have one or two fatty acid molecules attached at the ends. Approximately $1.2 \%$ is Free Astaxanthin (the same form as Synthetic Astaxanthin and Phaffia-derived Astaxanthin without any fatty acid molecules attached). The remaining $3.1 \%$ is a combination of other carotenoids including canthaxanthin, BC, zeaxanthin, and lutein (in descending order).

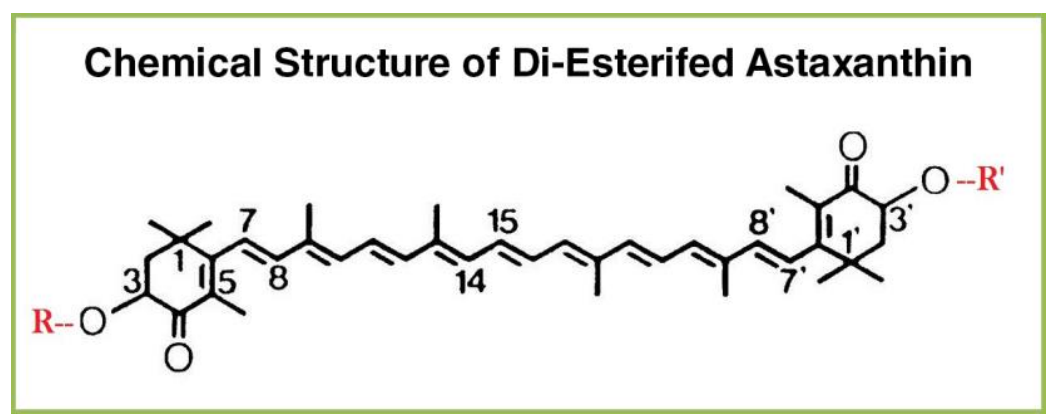

Figure 2. Natural Astaxanthin di-ester with one fatty acid molecule attached at each end of the Astaxanthin molecule, where R and R' are either 16:0 (Palmitic acid), 18:1 (Oleic acid), or 18:2 (Linolenic acid).

Difference \#2: The NAT-AX, SYN-AX and PH-AX molecules are shaped differently (Fig. 3). There are three distinct shapes known as enantiomers: Enantiomer one (known as "S"): 3S,3'S; Enantiomer two (known as "R"): 3R,3'R; Enantiomer three (known as “meso"): 3R,3'S.

- NAT-AX contains $100 \%$ "S" enantiomer 3S,3'S.

- SYN-AX contains a combination of three different enantiomers: It has $25 \% 3 \mathrm{~S}, 3$ 'S (the same molecular shape as NAT-AX). But it contains primarily molecules shaped differently than NAT-AX: $50 \%$ is meso-astaxanthin comprised of the $3 \mathrm{R}, 3$ ' $\mathrm{S}$ enantiomer and $25 \%$ is pure "R" enantiomer 3R,3'R.

- Finally, PH-AX is exclusively "R" enantiomer $3 R, 3$ 'R [6]. 


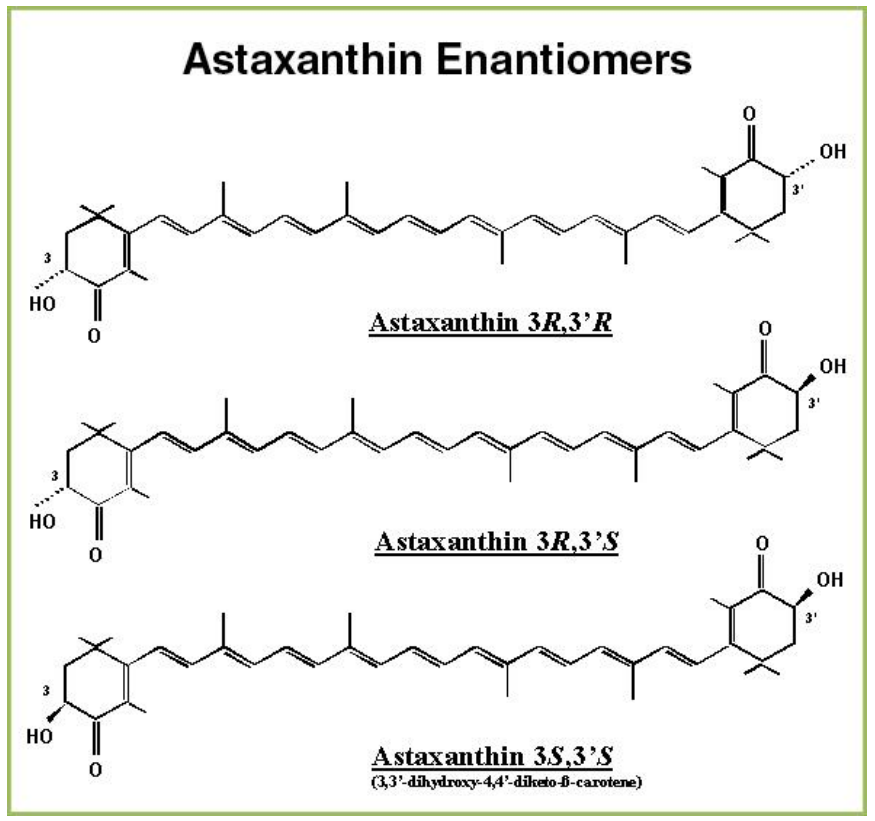

Figure 3. Although all three forms of Astaxanthin share the same molecular formula, they are shaped differently:

- Natural Astaxanthin from algae is exclusively 3S,3'S enantiomer.

- $100 \%$ of Phaffia-derived Astaxanthin molecules are shaped differently than Natural Astaxanthin with exclusively $3 \mathrm{R}, 3$ 'R enantiomer.

- $75 \%$ of Synthetic Astaxanthin molecules are shaped differently than Natural Astaxanthin molecules. Synthetic Astaxanthin contains a variety of all three enantiomers.

Difference \#3: SYN-AX and PH-AX are exclusively AX and contain no supporting carotenoids. In contrast, NAT-AX are naturally complex in Haematococcus pluvialis microalgae with other carotenoids present in small quantities. When lipids are extracted from the algae, the resulting extract contains primarily NAT-AX, but also contains four other naturally-occurring carotenoids (Fig. 1; Table 1) [7].

Table 1. Carotenoid Fractions in Astaxanthin from Algae

\begin{tabular}{|c|c|}
\hline $\begin{array}{r}\text { Breakdown of Carotenoid Fraction in } \\
\text { Natural Astaxanthin from Algae }\end{array}$ \\
\hline $87.01 \%$ & Mono-Esterified Astaxanthin \\
\hline $8.70 \%$ & Di-Esterified Astaxanthin \\
\hline $1.16 \%$ & Free Astaxanthin \\
\hline $0.95 \%$ & Canthaxanthin \\
\hline $0.83 \%$ & Beta-carotene \\
\hline $0.71 \%$ & Zeaxanthin \\
\hline $0.64 \%$ & Lutein \\
\hline $\mathbf{1 0 0 . 0 0 \%}$ & Total \\
\hline
\end{tabular}




\section{Differences in Antioxidant Activity between Forms of Astaxanthin}

Two head-to-head in-vitro studies comparing NAT-AX and SYN-AX have demonstrated far superior antioxidant activity of NAT-AX ranging from $14 \mathrm{X}$ more activity to as much as $90 \mathrm{X}$ more activity. To date, we're unaware of any antioxidant studies comparing NAT-AX with PH-AX; however, due to PH-AX being chemically more similar to SYN-AX (both are non-esterified, both have most or all R enantiomer, neither contains other carotenoids), we hypothesize that NAT-AX will also prove to be superior in antioxidant activity to PH-AX when tested head-to-head.

A study published in 2013 featured antioxidant testing as both university research at Creighton University (under the auspices of the prominent researcher Debasis Bagchi, $\mathrm{PhD}$ ) as well as independent laboratory analyses at Brunswick Laboratories. In these tests, NAT-AX was found to be a minimum of $14 \mathrm{X}$ stronger in antioxidant activity than SYN-AX. In the study at Creighton University, NAT-AX was tested for free radical elimination against SYN-AX as well as several other commonly used supplemental antioxidants. The study at Creighton University was performed in a Chronolog Lumivette luminometer to measure chemiluminescence as an index of reactive oxygen species production. In each case, NAT-AX was far more active with free radical elimination effects ranging from $14 \mathrm{X}$ greater than Vitamin $\mathrm{E}$ to $65 \mathrm{X}$ greater than Vitamin C. In the case of NAT-AX versus SYN-AX, the difference in antioxidant strength was greater than 20fold (Fig. 4; Table 2) [8].

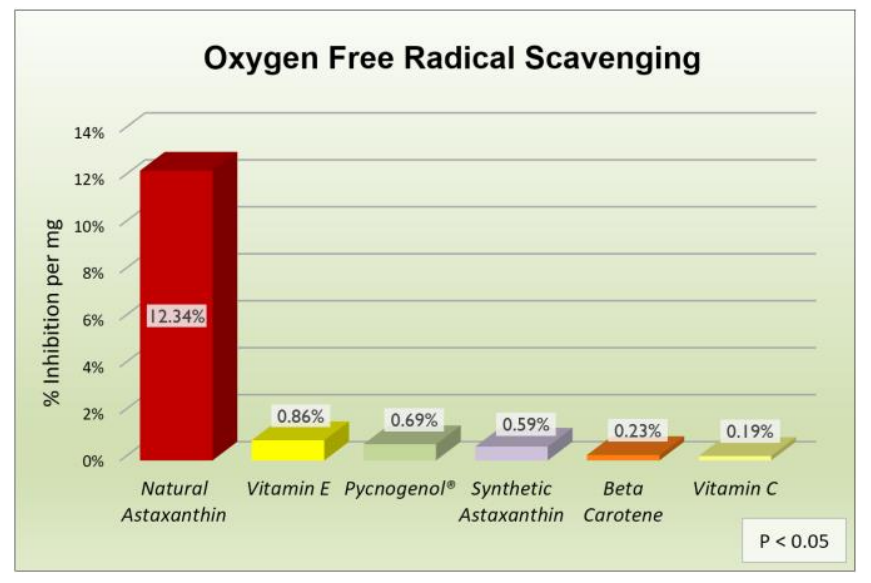

Figure 4. Natural Astaxanthin from algae showed far greater oxygen free radical eliminating activity than several other common antioxidants (ranging from 14X to 65X). In the case of Natural Astaxanthin versus Synthetic Astaxanthin, the difference was 21X.

Table 2. Difference in antioxidant strength

\begin{tabular}{|ccccc|}
\hline Table 2 & $\begin{array}{c}\text { mg of active } \\
\text { material used }\end{array}$ & $\begin{array}{c}\text { \% free radical } \\
\text { inhibition in study }\end{array}$ & $\begin{array}{c}\text { \% free radical } \\
\text { inhibition per mg } \\
\text { active material }\end{array}$ & $\begin{array}{c}\text { NAT-AX relative } \\
\text { performance }\end{array}$ \\
\hline Vitamin C & 100 & 19 & 0.19 & N-AX 65X stronger \\
\hline Vitamin E & 50 & 43 & 0.86 & N-AX I4X stronger \\
\hline Beta carotene & 100 & 23 & 0.23 & N-AX 53X stronger \\
\hline Pycnogenol & 100 & 69 & 0.69 & N-AX I8X stronger \\
\hline SYN-AX & 100 & 59 & 0.59 & N-AX 21X stronger \\
\hline NAT-AX & 5 & 61.7 & 12.34 & N/A \\
\hline
\end{tabular}


The independent lab testing performed at Brunswick Laboratories used Total ORACFN analyses which tests for five free radicals commonly found in the human body. Results were given as micromoles per Trolox Equivalent per gram. The in-vivo relevance of the Trolox Equivalent is that it is a means of evaluating comparative total antioxidant activity of pure substances and plant materials by an automated test. NAT-AX was many times more active in eliminating singlet oxygen, the superoxide ion and peroxyl radicals, while SYN-AX was more active in eliminating peroxynitrite. Results for NAT-AX were not determined for elimination of hydroxyl radicals, while SYN-AX obtained a positive result.

The comparison proffered by Brunswick Laboratories totaled the results for all five compounds and found that, overall, NAT-AX is $14 \mathrm{X}$ more active than SYN-AX. With regards to the harmful singlet oxygen radical, NAT-AX was 55X more active than SYN-AX (Table 3) [8].

Table 3. Antioxidant test results

\begin{tabular}{|c|c|c|c|}
\hline \multicolumn{4}{|c|}{ Brunswick Laboratories Antioxidant Test Results } \\
\hline Test & NAT-AX & SYN-AX & $\begin{array}{c}\text { NAT-AX VS. SYN- } \\
\text { AX }\end{array}$ \\
\hline Antioxidant power against singlet oxygen & 12,055 & 220 & $55 X$ stronger \\
\hline Antioxidant power against superoxide ion & 5,377 & 258 & $2 \mathrm{IX}$ stronger \\
\hline Antioxidant power against peroxyl radicals & 574 & 165 & $3.5 X$ stronger \\
\hline Antioxidant power against peroxynitrite & 28 & 115 & $0.24 X$ of SYN-AX \\
\hline Antioxidant power against hydroxyl radicals & Not Determined & 538 & Not Comparable \\
\hline 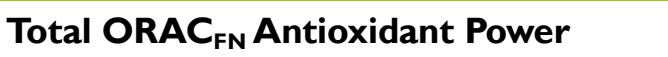 & 18,034 & 1,296 & I $4 X$ stronger \\
\hline
\end{tabular}

A recent study published by French university professors and sponsored by the French National Institute of Health and Medical Research again showed the superior antioxidant activity of NAT-AX compared to SYN-AX. This study was designed to examine the intracellular antioxidant capacity of these forms of $\mathrm{AX}$ as a means to understand potential cardiovascular benefits. The researchers tested two forms of NAT-AX against SYN-AX. The two natural forms tested were algae extracts produced by supercritical CO2 extraction and by solvent extraction using DMSO. The researchers did additional testing above and beyond a standard in-vitro antioxidant comparison in order to investigate the potential cardiovascular benefits for the different forms of Astaxanthin more deeply. They examined the intracellular antioxidant capacity of Astaxanthin using a stress-based model in HUVEC (human umbilical vein endothelial cells). The HUVEC cells were subjected to stress by introducing tert-butyl hydroperoxide (a molecule that increases intracellular production of ROS by damaging mitochondrial membranes). NAT-AX extracted by supercritical CO2 performed the best (although the difference with NAT-AX extracted with the solvent DMSO was not statistically significant). Both natural forms outperformed SYN-AX by tremendous margins ranging from $85 \mathrm{X}$ to $101 \mathrm{X}$ (Table 4). The researchers concluded that NATAX can inhibit intracellular-induced stress in human endothelial cells without toxicity. Since the intracellular antioxidant activity of NAT-AX was approximately $90 \mathrm{X}$ stronger than SYN-AX, they suggested that NAT-AX may have potential therapeutic or preventive properties for cardiovascular diseases [9]. 
Table 4. Antioxidant activity in SYN-AX, NAT-AX (DMSO extract and NAT-AX(CO2 extract)

\begin{tabular}{|ccc|}
\hline Product & $\begin{array}{c}\text { Cellular Antioxidant } \\
\text { Activity \% }\end{array}$ & $\begin{array}{c}\text { Comparative Increased Cellular } \\
\text { Antioxidant Activity (Using SYN- } \\
\text { AX as the base) }\end{array}$ \\
\hline SYN-AX & $0.3+/-0.2$ & N/A \\
\hline $\begin{array}{c}\text { NAT-AX } \\
\text { (DMSO extract) }\end{array}$ & $25.4+/-9.5$ & $85 X$ \\
\hline $\begin{array}{c}\text { NAT-AX } \\
\text { (CO2 extract) }\end{array}$ & $30.4+/-12.7$ & $101 X$ \\
\hline
\end{tabular}

Animal Research Shows Superior Efficacy and Bioactivity of NAT-AX in Comparison with SYN-AX and PH-AX

In addition to the in-vitro antioxidant studies cited above, pre-clinical research directly comparing the three sources of $\mathrm{AX}$ in different species of animals has clearly established the functional superiority of NAT-AX. In total, five animal trials have been published to date comparing AX sources; in each case, NAT-AX has demonstrated superior efficacy. Additionally, an innovative study comparing esterified and non-esterified forms of AX separated from Haematococcus pluvialis microalgae and tested in rats for inhibition of skin cancer and other health parameters has further corroborated these five animal trials; this study establishes a mechanism for the superior functioning of NAT-AX and is clear evidence that esterified forms of AX are more bioactive and provide enhanced health benefits than non-esterified AX.

\section{Survival Rates, Stress Resistance and Growth Rates in Shrimp}

The first study of health differences in animals supplemented with different forms of AX was done in 1998. It focused on a species of shrimp called Penaeus monodon (known as the "giant tiger prawn"). This study was done by university researchers in Thailand in support of the large shrimpfarming industry in that country. These professors did a series of tests on three different larval and post-larval stages during the shrimp's life cycle. They separated the shrimp into four different groups:

- One treatment group was fed a commercial diet supplemented with NAT-AX.

- The second treatment group was fed a commercial diet supplemented with SYN-AX.

- One control group was fed the same commercial diet without any addition of AX.

- A different control group was fed a natural diet that the shrimp would normally eat in the wild.

Fifteen days after the post-larval stage, shrimp fed NAT-AX were experiencing significantly better survival rates than all three other groups (including shrimp fed the natural diet). And in each of the three life stages, shrimp fed NAT-AX survived at higher rates than shrimp fed SYN-AX. (Remarkably, in the zoea larval stage, $82.5 \%$ of the shrimp fed NAT-AX survived while only $27.8 \%$ of the shrimp fed SYN-AX survived.) The differences were statistically significant between NAT-AX and SYN-AX in both other life stages, albeit with less dramatic results (Table 5). 
Table 5. Different diets impact on survival rate of shrimp

\begin{tabular}{|cccc|}
\hline & \multicolumn{3}{c|}{ Survival of larval stage (\%) } \\
\hline Diets & Zoea & Mysis & Postlarvae \\
\hline Natural Diet & $82.0 \pm 3.30$ & $76.7 \pm 8.61$ & $55.2 \pm 6.14$ \\
\hline $\begin{array}{c}\text { Commercial Diet without } \\
\text { Astaxanthin added }\end{array}$ & $74.0 \pm 5.19$ & $57.3 \pm 5.01$ & $68.6 \pm 5.73$ \\
\hline $\begin{array}{c}\text { Commercial Diet with Algae- } \\
\text { based Astaxanthin added }\end{array}$ & $82.5 \pm 3.53$ & $69.7 \pm 12.05$ & $76.0 \pm 9.46$ \\
\hline $\begin{array}{c}\text { Commercial Diet with Synthetic } \\
\text { Astaxanthin added }\end{array}$ & $27.8 \pm 4.01$ & $58.1 \pm 0.29$ & $64.4 \pm 11.86$ \\
\hline
\end{tabular}

Additionally, tests of low water salinity were done to examine the different groups' tolerance levels to environmental stress, and the shrimp fed the NAT-AX diet again outperformed all others. Shrimp from all three other groups died faster than the NAT-AX fed shrimp when subjected to this stressful condition (Fig. 5).

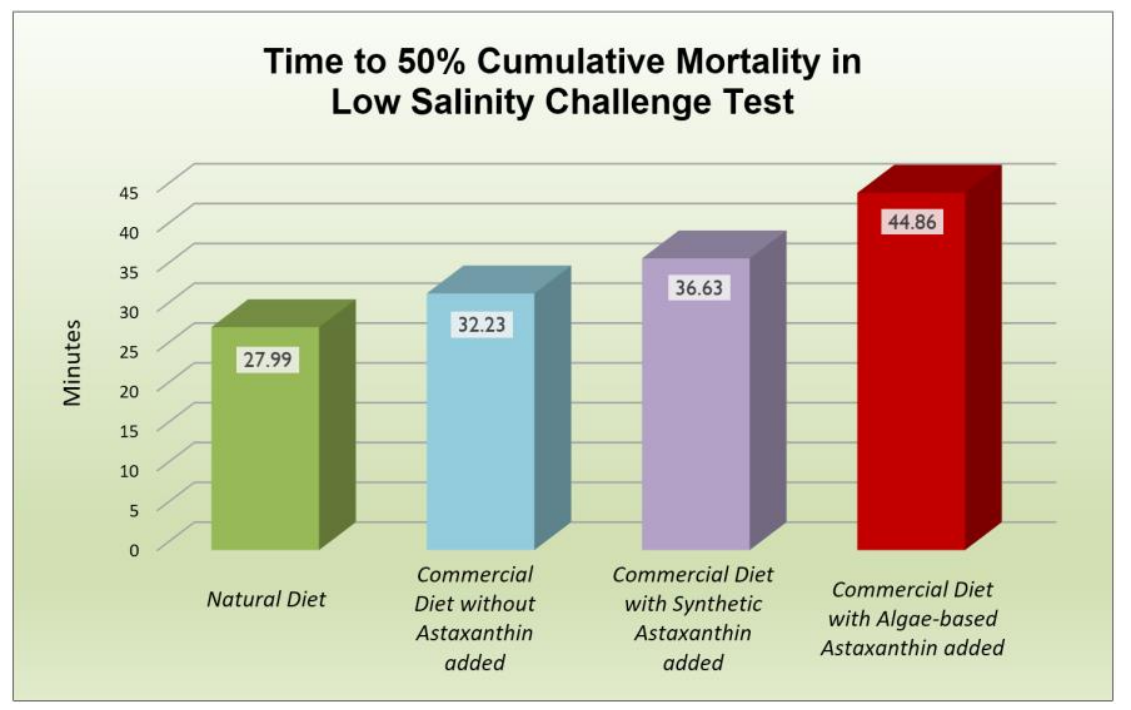

Figure 5. Shrimp larvae subjected to environmental stress survived longer when fed a diet containing Natural Astaxanthin from algae compared to all three other diets.

Finally, there were differences in growth rates as well between diets. Measured at 15 days after the larval stage, shrimp fed the NAT-AX diet were larger on average than all other groups. In each case, the result was statistically significant (Table 6).

Table 6. Different diets impact on length of post larval shrimp

\begin{tabular}{|cc|}
\hline Diets & $\begin{array}{c}\text { Length of Post Larval Shrimp } \\
(\mathrm{cm})\end{array}$ \\
\hline Natural Diet & $0.9843 \pm 0.073$ \\
\hline $\begin{array}{c}\text { Commercial Diet without Astaxanthin } \\
\text { added }\end{array}$ & $0.9674 \pm 0.074$ \\
\hline $\begin{array}{c}\text { Commercial Diet with Algae-based } \\
\text { Astaxanthin added }\end{array}$ & $1.0019 \pm 0.067$ \\
\hline $\begin{array}{c}\text { Commercial Diet with Synthetic } \\
\text { Astaxanthin added }\end{array}$ & $0.9652 \pm 0.075$ \\
\hline
\end{tabular}


It's interesting to note that shrimp fed SYN-AX were generally the worst performers. Most notably, in the Zoea larval stage, all three other groups outlived the shrimp fed SYN-AX by vast margins. This is particularly surprising when considering that one of the control diets was precisely the same commercial diet contained in the NAT-AX and SYN-AX diets, but without any AX added.

The researchers hypothesized that shrimp accept NAT-AX better than SYN-AX, which is why the shrimp supplemented with NAT-AX survive at much higher rates, grow faster, and resist environmental stress more efficiently. They cited the difference in esterification (NAT-AX being predominantly esterified and SYN-AX being non-esterified "Free Astaxanthin") as the probable basis for NAT-AX outperforming SYN-AX [10].

This study's results are substantial evidence for the superiority of NAT-AX over SYN-AX in this species due to the variety of tests run and the consistency of results. They tested differences at multiple life stages (two larval stages as well as 15 days into the post-larval stage) and they tested effects in both healthy environments as well as under environmental stress. In all cases, NAT-AX outperformed SYN-AX.

\section{Gastric Ulcers in Rats-Study \#1}

A study done in 2005 at a university in Japan examined the effects of AX on gastric ulcers in rats. Results indicated significantly better potential for NAT-AX to prevent gastric ulcers than both PHAX and SYN-AX. Rats were stressed by putting them into chest-level water for 24 hours after having fasted for 24 hours. This study tested the three forms of AX as well as beta-carotene. All the rats given carotenoids (including all three forms of $\mathrm{AX}$ as well as beta-carotene) before being stressed were appreciably protected against the formation of gastric ulcers as compared to rats in the control group. The rats given NAT-AX experienced improved health over the other groups as evidenced by statistically significant smaller ulcer indexes. The researchers theorized that AX improves antitumor immune response through prevention of lipid peroxidation induced by stress [11].

\section{Gastric Ulcers in Rats-Study \#2}

In 2008, a similar study was done on the effects of AX on ulcers in rats. This study tested SYNAX against NAT-AX, but did not include PH-AX. The researchers used ethanol to induce ulcers and found that pre-treatment with NAT-AX outperformed SYN-AX in inhibiting enzymes associated with the formation of ulcers. SYN-AX did not show any inhibition all. Remarkably, NAT-AX showed inhibition of ulcers at a higher level than the ulcer drug omeprazole (as estimated from other studies. Note: Omeprazole is sold under the brand name Prilosec ${ }^{\circledR}$ ). NAT-AX showed gastroprotective effects dose-dependently on ethanol-induced gastric lesions. The researchers pointed out that NAT-AX showed better stability than Free AX (the form found in SYN-AX), and hypothesized that this may be the basis for its superior health benefits [12]. The better stability is likely due to esterified AX being more stable than free AX.

\section{Antioxidant Activity and Increase of Lifespan in Model Organism for Longevity in Mammals}

A recent study sponsored by the Chinese government's National Natural Science Foundation tested all three forms of AX in a model organism for longevity testing in mammals to see if $\mathrm{AX}$ can increase lifespan. This study was published as a joint project between the Department of Food 
Science at University of Massachusetts and the Department of Food Science at South China Agricultural University. The model organism is a worm named Caenorhabditis elegans (C. elegans). This worm is extensively used in longevity testing for two strategic reasons: Firstly, it has $60 \%$ to $80 \%$ of the human gene homologues (linking experiments with this worm to potential increase in longevity in humans) [13]. Secondly, this worm has a three week lifespan, allowing for rapid testing and results.

The worms were separated into four groups: A control group, a group treated with NAT-AX, a group treated with PH-AX, and a group treated with SYN-AX. Similar to our goal with this review paper, the goal of this worm study was to test functional differences between the different forms of AX to see if there is a preferred form for human supplementation.

The worms underwent oxidative stress for 24 hours. The researchers created oxidative stress by introducing paraquat (a toxic, fast-acting herbicide) to the worms. The worms were tracked for five days after exposure to paraquat. Reactive oxygen species were reduced by all forms of AX, with NAT-AX being significantly superior in quenching these free radicals, particularly versus SYN-AX in which it was over $80 \%$ more effective (Fig. 6).

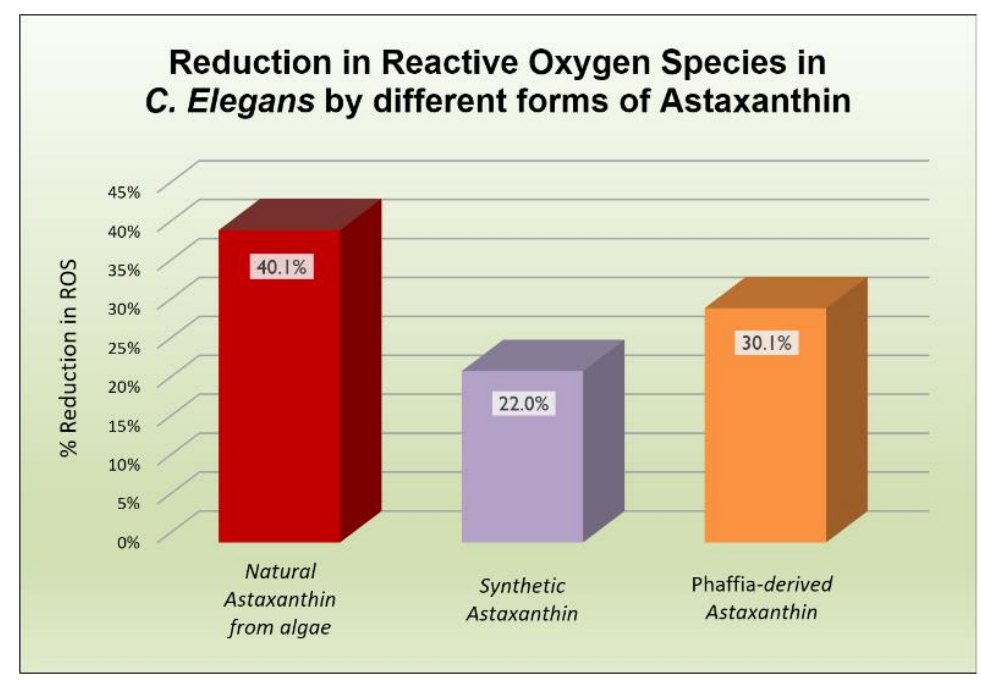

Figure 6. Natural Astaxanthin from algae eliminated ROS much more effectively than Synthetic Astaxanthin (82.3\% more effective) and Phaffia-derived Astaxanthin (33.2\% more effective).

NAT-AX steadily increased survival rates of the worms at all five measurement intervals versus control. By Day 3, the survival rates were $19 \%$ better than control, and by Day 5, survival rates were $110 \%$ better. NAT-AX outperformed SYN-AX and PH-AX with regards to survival rates slightly; however, contrary to the profound differences in shrimp larvae cited above, in this study the differences were not statistically significant. The researchers concluded that AX can increase oxidative resistance, decrease levels of reactive oxygen species, increase enzyme activity of superoxide dismutase (SOD) and Catalase, and enhance expression of SOD-3 (a gene that encodes SOD), all of which lead to increasing survival of the worms [14].

\section{Endurance in Mice}

This is the most recent study comparing the effects of NAT-AX to other forms in animals. The mice in this study were divided into four groups: Control; mice fed NAT-AX; mice fed SYN-AX; and mice fed PH-AX. 
The study duration was five weeks, during which supplementation of the various AX forms was consistently administered. At the end of five weeks of supplementation, the mice were run until exhaustion on a treadmill. Mice fed NAT-AX ran significantly longer than mice fed both other AX forms (Fig. 7).

Additional differences between the groups were noted:

- Plasma concentration of AX was significantly higher in the NAT-AX group than all other groups

- Similarly, tissue concentration of AX was significantly higher in the NAT-AX group than all other groups

- NAT-AX significantly increased 5'- adenosine monophosphate-activated protein kinase (AMPK) levels in skeletal muscle

- Although mice in the NAT-AX group ran for a longer time, hexanoyl lysine (HEL) adduct levels in skeletal muscle mitochondria were similar in the control and NAT-AX groups

The researchers hypothesized that accumulation of $\mathrm{AX}$ in muscle tissue may increase endurance. They investigated energy metabolism and oxidative damage in order to understand the mechanisms involved in increasing endurance. AMPK has substantial influence in regulating metabolism of both carbohydrates and lipids. Furthermore, AMPK is responsible for increasing mitochondrial biosynthesis and activity. NAT-AX significantly increased AMPK levels, which they attributed to the increase in running endurance due to activation of energy metabolism.

HEL is a marker used to measure oxidative stress which triggers the initial phase of lipid peroxidation. NAT-AX inhibited the increase in HEL caused by endurance exercise in mice in this study. NAT-AX was successful in preventing oxidative damage in skeletal muscle mitochondria. This may be due to suppression of lactic acid levels. Previous clinical research has found NATAX capable of reducing lactic acid levels in athletes after running [15]. Although lactic acid levels were not measured in this study, the authors suggested a possible correlation and offer lactic acid suppression as another possible mechanism. In fact, they proffered that, due to NAT-AX's variety of functions, its effects on exercise performance may involve various other mechanisms. Their conclusion stated that NAT-AX promotes energy production and protects tissue from oxidative damage during exercise. They attribute these results to the esterified form (NAT-AX) having superior absorption characteristics than non-esterified forms (SYN-AX and PH-AX) [16].

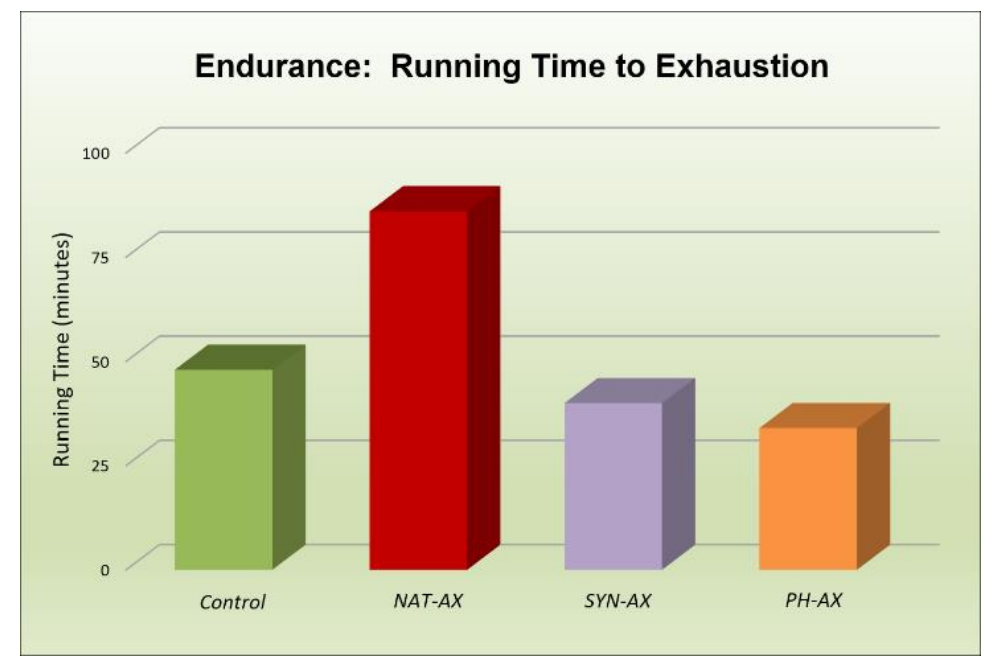

Figure 7. NAT-AX significantly increased endurance in mice as measured by time to exhaustion on a treadmill, while SYN-AX and PH-AX decreased endurance as compared to the control group. 


\section{Skin Cancer, Antioxidant Activity, Retinol Levels and Tyrosinase Enzyme Levels in Rats}

This unique study was sponsored by the government of India. The study employed an alternate means to obtain the different forms of AX than the previously cited studies. In each of the five animal trials referenced above (as well as the antioxidant surveys), SYN-AX was obtained from petrochemicals as Free Astaxanthin (FREE-AX); PH-AX was obtained from geneticallymanipulated yeast as FREE-AX; and NAT-AX was obtained from Haematococcus pluvialis microalgae as predominantly Esterified AX (EST-AX). In this study, the researchers extracted the total carotenoid fraction from Haematococcus pluvialis and then separated the fractions into FREE-AX, mono-esterified AX, and di-esterified AX. This process separated the algae extract into the FREE-AX form which is exclusively found in SYN-AX and PH-AX and two different esterified forms that are the main components of NAT-AX. They analyzed these three forms along with samples of the total carotenoid fraction before separation. Rats were fed at 100 or 200 micrograms per KG of each form for 14 days, after which the rats in the treatment group were exposed to UV and DMBA to induce skin cancer on Day 15 (exposure continued on a daily basis until Day 60).

A crucial finding of this study indicated that EST-AX from Haematococcus pluvialis microalgae inhibited skin tumors in rats significantly better than FREE-AX (the form found in SYN-AX and PH-AX) (Figure 8). Additionally, EST-AX had far superior antioxidant activity compared to FREE-AX (Figure 9); increased retinol conversion in the liver more efficiently; and augmented tyrosinase enzyme more successfully (Figure 10). The conclusion stated that EST-AX from algae has better anti-cancer potential than FREE-AX (as found in SYN-AX and PH-AX) which may be due to better bioavailability [17].

This study, while not done on SYN-AX synthesized from petrochemicals or PH-AX derived from mutated yeast, is perhaps the most significant examination of the effect of esterification of AX to date. While all three forms of AX in this study were derived from Haematococcus pluvialis microalgae, they were separated into different esterified and non-esterified forms. Results indicated that the esterified forms show far superior therapeutic and preventive health potential than the non-esterified form. Since the source of all forms was the same (Haematococcus pluvialis microalgae), other variables possible in commercial AX products were eliminated. Hence, the superiority of EST-AX over FREE-AX in this study can only be attributed to the presence of esters. This is a significant factor in our conclusion that EST-AX from algae is clearly the superior choice for human supplementation.

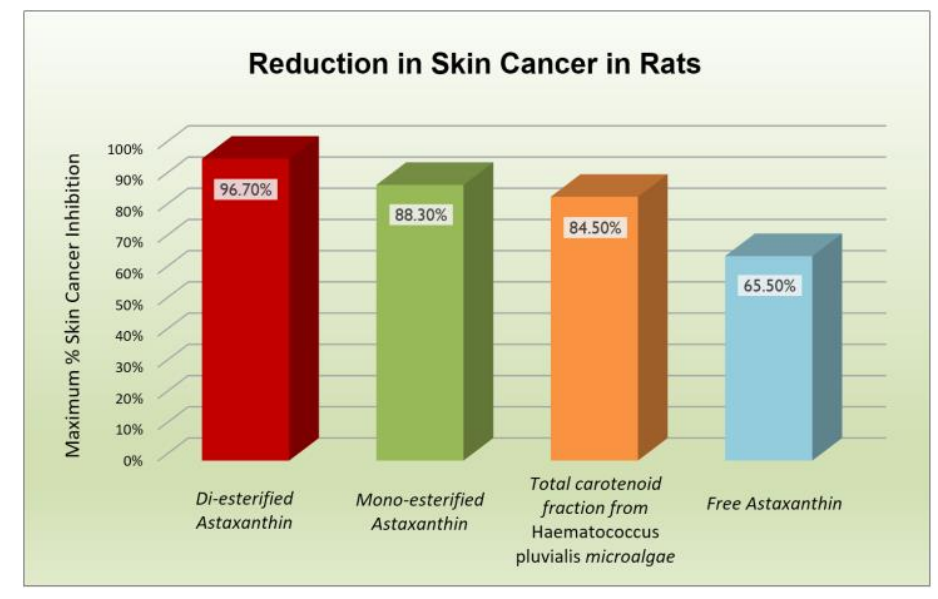

Figure 8. Both di-esterified NAT-AX and mono-esterified NAT-AX inhibited skin cancer in 
rats more effectively than Free-AX and total carotenoid fraction from Haematococcus pluvialis microalgae. In the case of di-esterified NAT-AX, inhibition reached a level of $96.7 \%$.

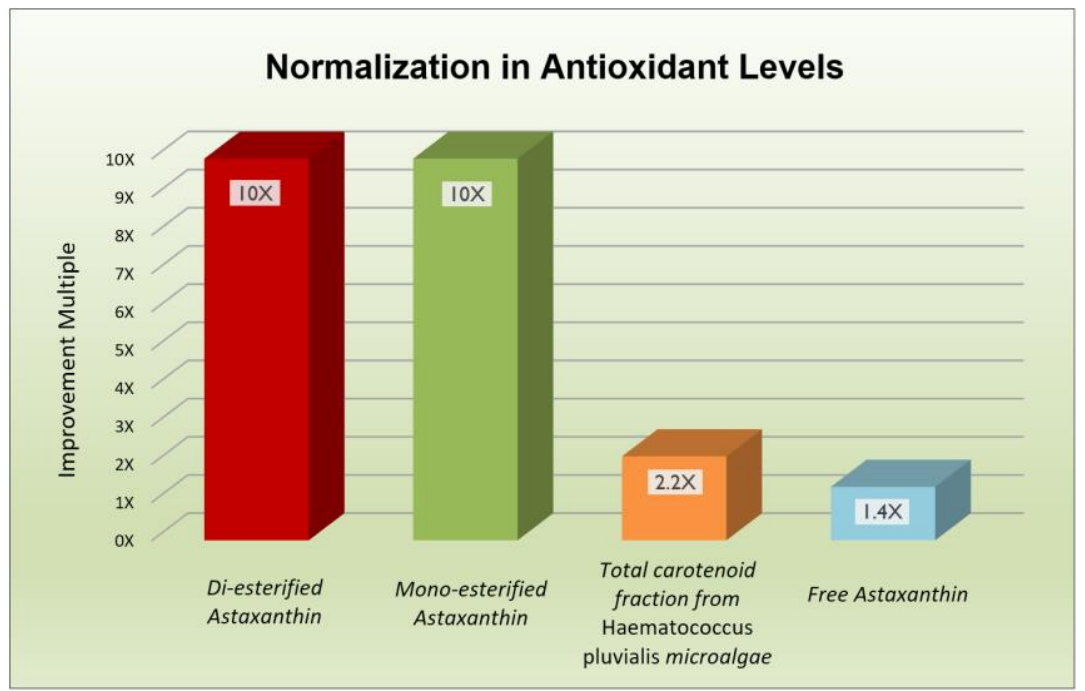

Figure 9. Esterified forms of Astaxanthin from microalgae had far superior antioxidant activity compared to Free Astaxanthin and Total Carotenoids from Haematococcus pluvialis microalgae.

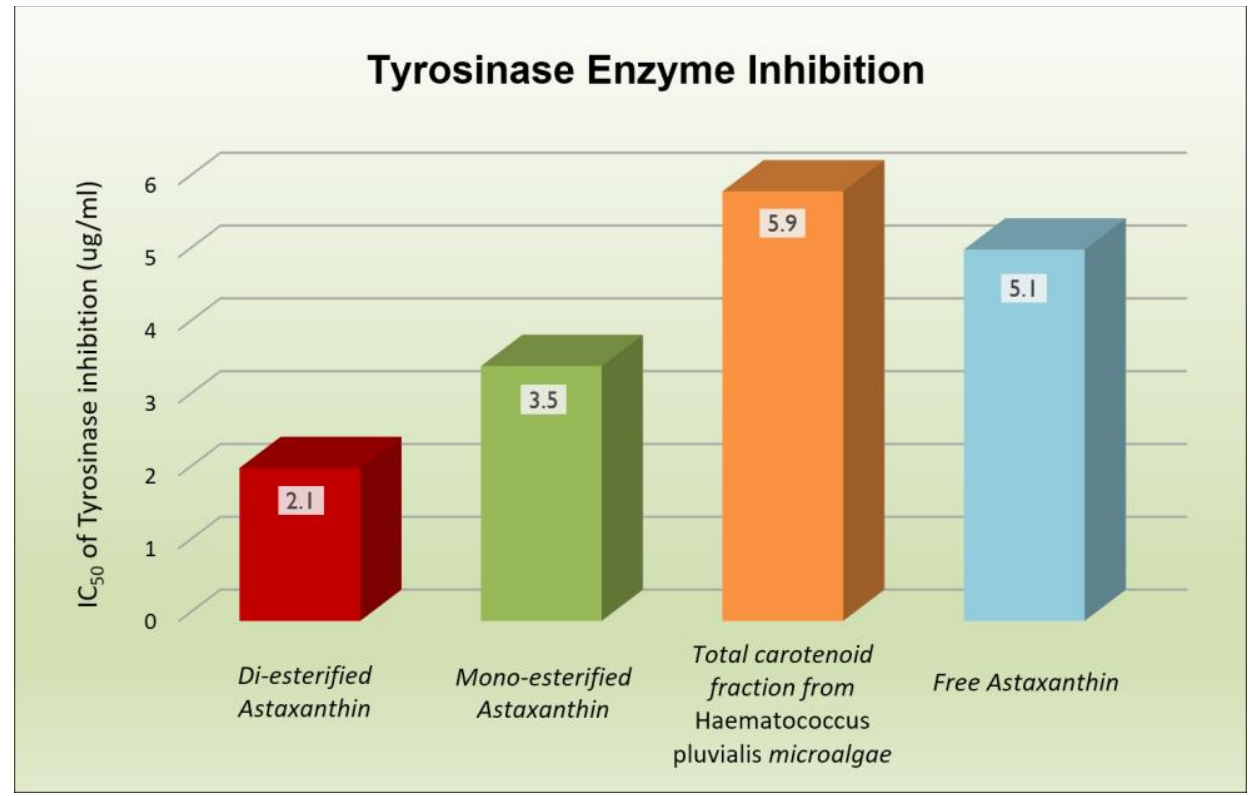

Figure 10. Di-esterified AX had 2.4X - 2.8X less tyrosinase enzyme inhibitory activity than Free Astaxanthin and Total Carotenoids from Haematococcus pluvialis microalgae (respectively), while Mono-esterified AX had 1.5X - 1.7X less inhibitory activity (respectively). All results were statistically significant. Tyrosinase enzyme is located in melanocytes which produce melanin. Tyrosinase is responsible for the first step in melanin production.

\section{CONCLUSIONS}

NAT-AX is the logical choice as a consumer dietary supplement amongst forms of AX currently in the market as evidenced in this review of the literature. The majority of the comparative studies cited above were sponsored by and/or conducted by governmental agencies and universities; as 
such, they are likely to be more impartial than studies sponsored by AX suppliers and free of conflicts of interest.

NAT-AX has proven far more active as an antioxidant than SYN-AX by a factor 14X to 90X. Due to the chemical similarities between PH-AX and SYN-AX, we postulate that NAT-AX will prove far more active than PH-AX as well if tested in head-to-head antioxidant surveys.

However, in-vitro antioxidant testing does not necessarily equate to corporal actuality in humans. While human clinical trials directly comparing forms of AX for safety and health benefits would be the determining factor, no such research has been conducted to date. In fact, there is a complete dearth of research in humans for SYN-AX and PH-AX demonstrating efficacy or even safety. This is a huge concern for SYN-AX and PH-AX - we don't currently know if they have health benefits for humans or if they are completely safe for human consumption on a long-term basis.

Due to the lack of human clinical research for SYN-AX and PH-AX, we turn to animal research directly comparing the three sources to better understand their potential as human dietary supplements for preventive or therapeutic effects. Five animal trials in different animal species directly comparing different forms of AX have undisputedly demonstrated that NAT-AX from algae is the most effective form for a variety of health benefits. This research has established that NAT-AX is superior in:

- Increasing survival rates

- Improving resistance to environmental stress

- Improving exercise endurance

- Prevention of gastric ulcers

- Expanding antioxidant activity

- Increasing growth rates

Additionally, a unique study in rats comparing esterified and non-esterified forms provides weighty evidence that NAT-AX is superior to SYN-AX and PH-AX — and this study gives a basis for why this is so. By separating esterified and non-esterified AX from a single sourceHaematococcus pluvialis microalgae - the researchers conducting this study eliminated other potential complicating factors that could surface by using AX from different sources. They definitively demonstrated the superiority of Esterified Astaxanthin (the form present in NAT-AX) over Free Astaxanthin (the form present in SYN-AX and PH-AX) by demonstrating that esterified AX is superior to Free Astaxanthin at:

- Preventing the formation of skin cancer

- Amplifying antioxidant activity

- Significantly augmenting retinol conversion in the liver

- Increasing tyrosinase enzyme levels

This innovative study establishes one mechanism by which NAT-AX consistently outperforms SYN-AX and PH-AX in the studies cited in this review-Esterification. The two additional chemical differences between AX forms (stereochemistry and presence of other carotenoids) may also play a role in the functional superiority of NAT-AX, but at present, these factors have not been isolated and tested. Additionally, possible explanations for the functional superiority of NAT-AX proffered in three of the animal trials cited above (improved bioavailability 
of NAT-AX and better stability of NAT-AX) may also be proven to be true; we hope to see research in this area in the future.

Due to the nonexistence of clinical trials showing health benefits and the complete absence of direct safety research in humans for SYN-AX and PH-AX, we highly recommend that consumers supplement with NAT-AX as the sole clinically-validated form. NAT-AX has proven safe and effective over many years of consumer use. NAT-AX has amassed an abundance of safety data and has demonstrated extensive health benefits in many human clinical studies. These factors have led regulatory bodies throughout the world to accept NAT-AX for direct human consumption in supplement form. Owing to the lack of such research, SYN-AX and PH-AX have only been accepted in a few countries to date.

As a result of the preponderance of evidence above, we recommend that consumers supplementing with AX ensure that the brand of AX they consume sources raw materials from Haematococcus pluvialis algae.

List of Abbreviations: AX: Astaxanthin. NAT-AX: Natural Astaxanthin from Haematococcus pluvialis microalgae. SYN-AX: Synthetic Astaxanthin from petrochemicals. PH-AX: Astaxanthin from the genetically-manipulated yeast Xanthophyllomyces dendrorhous (former nomenclature Phaffia rhodozyma, still commonly referred to as "Phaffia"). EST-AX: Esterified Astaxanthin. FREE-AX: Free Astaxanthin. BC: Beta-Carotene. ROS: Reactive oxygen species. HUVEC: Human umbilical vein endothelial cells. C. Elegans: Caenorhabditis elegans. SOD: Superoxide dismutase. AMPK: 5' - adenosine monophosphate-activated protein kinase. HEL: Hexanoyl lysine

Competing Interests: Bob Capelli and Lixin Ding, PhD work for companies involved in the production of Natural Astaxanthin from microalgae. Shawn Talbott, PhD is the owner of an independent clinical research organization and is not involved in the production of Astaxanthin.

Authors' Contributions: All authors contributed equally to this manuscript.

Acknowledgements and Funding: While Bob Capelli and Lixin Ding work for companies involved in the production of Astaxanthin from microalgae, this manuscript was written independently of their responsibilities at those companies. No funding for this review was given by any company or other entity.

\section{REFERENCES}

1. Kidd P: Astaxanthin, cell membrane nutrient with diverse clinical benefits and anti-aging potential. Altern Med Rev 2011, 16(4):355-364.

2. Fassett R, Coombes J: Astaxanthin, oxidative stress, inflammation and cardiovascular disease. Future Cardiol 2009, 5(4):333-342.

3. Guerin M, Huntley M, Olaizola M: Haematococcus Astaxanthin: applications for human health and nutrition. Trends Biotechnol 2003, 21(5):210-216.

4. McNulty H, Jacob RF, Mason RP: Biologic activity of carotenoids related to distinct membrane physicochemical interaction. Am J Cardiol 2008, 101(10A):20D-29D. 
5. Beutner S, Bloedorn B, Frixel S, Blanco I, Hoffmann T, Martin H, Mayer B, et al.: Quantitative assessment of antioxidant properties of natural colorants and phytochemicals: carotenoids, flavonoids, phenols and indigoids. The role of B-carotene in antioxidant functions. J Sci Food Agric 2001, 81(6):559-568.

6. Visioli F, Artaria C: Astaxanthin in cardiovascular health and disease: mechanisms of action, therapeutic merits, and knowledge gaps. Food Funct 2017, 8(1):39-63.

7. Craft Technologies Independent Laboratory Report, November 2015. On file at BGG North America, Irvine, CA, USA.

8. Capelli B, Bagchi D, Cysewski G: Synthetic Astaxanthin is significantly inferior to algalbased Astaxanthin as an antioxidant and may not be suitable as a human nutritional supplement. NutraFoods 2013, 12(4):145-152.

9. Regnier P, Bastias J, Rodriguez-Ruiz V, Caballero-Casero N, Caballo C, Sicilia D, Fuentes A, et al.: Astaxanthin from Haematococcus pluvialis prevents oxidative stress on human endothelial cells without toxicity. Mar Drugs 2015, 13(5):2857-2874.

10. Darachai J, Piyatiratitivorakul S, Kittakoop P, Nitihamyong C, Menasveta P: Effects of Astaxanthin on Larval Growth and Survival of the Giant Tiger Prawn, Penaeus monodon. In Advances in shrimp biotechnology: Proceedings to the Special Biotechnology, 5th Asian Fisheries Forum: 11-14 November 1998; Chiengmai, Thailand. Edited by Flegel TW. National Center for Genetic Engineering and Biotechnology; 1998:117-122.

11. Nishikawa Y, Minenaka Y, Ichimura M, Tatsumi K, Nadamoto T, Urabe K: Effects of astaxanthin and vitamin $\mathrm{C}$ on the prevention of gastric ulcerations in stressed rats. J Nutr Sci Vitaminol (Tokyo) 2005, 51(3):135-141.

12. Kamath B, Srikanta B, Dharmesh S, Sarada R, Ravishankar G: Ulcer preventive and antioxidative properties of astaxanthin from Haematococcus pluvialis. Eur J Pharmacol 2008, 590(1-3):387-395.

13. Kaletta T, Hengartner M: Finding function in novel targets: C. elegans as a model organism. Nat Rev Drug Discov 2008, 5(5):387-399.

14. Liu X, Luo Q, Cao Y, Goulette T, Liu X, Xiao H: Mechanism of different stereoisometric Astaxanthin in resistance to oxidative stress in Caenorhabditis elegans. J Food Sci 2016, 81(9):H2280-H2287.

15. Sawaki K, Yoshigi H, Aoki K, Koikawa N, Azumane A, Kaneko K, Yamaguchi M: Sports performance benefits from taking Natural Astaxanthin characterized by visual acuity and muscle fatigue improvement in humans. J Clin Ther Med 2002, 18:10851100 .

16. Aoi W, Maoka T, Abe R, Fujishita M, Tominaga K: Comparison of the effect of nonesterified and esterified Astaxanthins on endurance performance in mice. J Clin Biochem Nutr March 2018, 62(2):161-166.

17. Rao A, Sindhuja H, Dharmesh S, Sankar K, Sarada R, Ravishankar G: Effective inhibition of skin cancer, tyrosinase, and antioxidative properties of astaxanthin and astaxanthin esters from the green alga Haemaotococcus pluvialis. J Agric Food Chem 2013, 61(16):3842-3851. 